\title{
Signal Timing Optimization for Transit Priority at Near-Saturated Intersections
}

\author{
Shijie Shu, Jing Zhao $(\mathbb{D}$, and Yin Han \\ Department of Traffic Engineering, University of Shanghai for Science and Technology, Shanghai 200093, China \\ Correspondence should be addressed to Jing Zhao; jing_zhao_traffic@163.com
}

Received 28 February 2018; Revised 2 June 2018; Accepted 3 July 2018; Published 11 July 2018

Academic Editor: Alain Lambert

Copyright (C) 2018 Shijie Shu et al. This is an open access article distributed under the Creative Commons Attribution License, which permits unrestricted use, distribution, and reproduction in any medium, provided the original work is properly cited.

\begin{abstract}
Transit signal priority is a useful way to improve transit operations in urban networks. Most of the existing studies have been conducted in conditions with low saturation to avoid the detrimental effects of vehicles without priority. However, from the public transit point of view, it is more meaningful to assign transit signal priority when the degree of the saturation intersections is high. This study proposes a signal control model for transit signal priority to minimize the overall delay at near-saturated intersection. The delay increment is calculated in three scenarios for buses and private vehicles according to the dissipation time of the vehicular queue. A set of constrains are set up to avoid queue overflows and to ensure the rationalization of the signal timing. The proposed control model is tested based on a case study and numerical experiments. The results show that the proposed model can reduce the total person delay at near-saturated intersections. The length of priority time, degree of saturation, and number of lanes are the three main influencing factors. More than $6 \%$ reductions in person delay can be obtained for undersaturated intersections when the priority time is less than $5 \mathrm{~s}$. Moreover, even when the intersection saturation is 0.95 , the bus signal priority can be applied if only the priority time is less than $5 \mathrm{~s}$.
\end{abstract}

\section{Introduction}

As cities become more and more congested, land for road construction is limited. Therefore, congestion needs to be alleviated by changing the model of travel [1]. The improvement of public transit service can elicit important social benefits. Transit signal priority and exclusive bus lanes are two common methods used to improve the public transit service [2]. Setting exclusive bus lanes is a cost-effective approach for providing a high-quality transit service, enhancing the reliability of bus services, and increasing the speed of buses [3-6]. Although continuous running ways yield great benefits to transit operations, transit vehicles will be delayed at intersections by traffic signals [7], especially buses that arrive at the end of the green light period. Therefore, to further improve the running efficiency of buses at intersections, the exclusive bus lanes need to be perfected by assigning transit signal priority.

Transit signal priority is a typical strategy used to reduce the delay of public transit, and it has been used extensively to improve transit operations in urban networks. Existing transit signal priority strategies can be divided into three major categories: passive priority strategies, active priority strategies, and real-time priority strategies [8].

Passive priority strategies are developed on the basis of historical data and do not require any detection system. Skabardonis introduced transit priority measures on urban networks [9]. It has been proven that the passive priority strategies are effective in simple network configurations and elicit predictable dwell times and high bus frequencies. However, it was also pointed out that the passive priority strategies were less adaptable when vehicular flow was low. Estrada proposed a passive signal priority simulation model which restricted the maximum car passenger delay increment [10]. The model was applied to a series of trial networks, and the results showed that the model can reduce the bus travel times by $8.5 \%$, while concurrently increasing the car delay by $5 \%$. Correspondingly, the detection system developed numerous active priority strategies which could provide more effective priority to buses.

Active priority strategies include phase extension, phase advance, phase insertion, and phase rotation [11]. These 
strategies respond to traffic variations in real-time and are therefore more effective than passive priority strategies. Ngan et al. examined the impacts of a set of traffic parameters on the effectiveness of a transit signal priority application [12]. The results show that transit signal priority application would be more effective when the bus volume is moderate to heavy. Jacobson and Sheffi presented an analytical model for the bus preemption analysis [13]. The results showed that the benefits of bus preemption were small when the cross-traffic flow was much lower than the preemption direction. Furth and Muller put forward a method of operational control which can keep buses on schedule [14]. This method has been implemented in the Netherlands. The results showed that the proposed method can improve the bus schedule. In addition, conditional priority elicited minimal delay increments compared to the case where no priority was assigned, while absolute priority caused large delay increments. Zeeshan et al. proposed an analytical model for estimating the delay impacts of several transit priority strategies [15]. Active priority strategies often have detrimental effects on the vehicles that have no priority, so real-time priority strategies were developed which focused on the total vehicular delay or the total passenger delay at signal intersections.

Real-time priority strategies optimize signal timings based on performance criteria, such as passenger delays, vehicular delays, and combinations of vehicular delays. Ling and Shalaby put forward an algorithm which is based on reinforcement learning and the control policy is learned by the algorithm [16]. The best duration of each phase is determined by the reinforce learning. The experimental results show that the proposed algorithm can reduce the transit headway deviation and disrupt cross street traffic smaller. Christofa and Skabardonis presented a real-time signal control system for signal priority on conflicting transit routes that also minimized the negative effects on vehicular traffic [17]. To solve the issue of multiple priority requests, Head proposed a model which could analyze complex controller behaviors [18]. The analyzed results showed that the proposed model for serving priority requests resulted in fewer delays than a first-come, first-served policy. From the perspective of intersection groups, Ma presented an optimization model which could generate the optimal combination of priority strategies for intersection groups [19]. Christofa et al. presented a realtime signal control system for assigning signal priority on conflicting transit routes that also minimized the passenger delay on arterials [20]. Ma et al. proposed a priority signal model for multiple bus requests [21]. The optimal priority serving sequence is generated by the proposed model, so the bus can maximize the utilization of available green times. With the development of communication technologies, optimization methods were proposed in a connected vehicle environment. Hu presented an optimization method for an intelligent transit signal priority logic that enables bus signal cooperation among consecutive signals [22]. The results showed that the intelligent TSP logic can efficiently reduce the bus delay compared to the conventional TSP. Li et al. proposed an adaptive transit signal priority optimization model that optimizes green splits for three consecutive cycles to minimize the vehicle delay [23]. The numerical example results show that the bus delay and the traffic delay along the bus movement direction were all reduced. He and Head presented a request-based, mixed-integer, linear program, which can coordinate multiple priority requests from pedestrians and different modes of vehicles [24]. Lin presented a control model that minimized bus passenger waiting time at downstream bus stops but did not increase the delay for vehicular passengers [25]. The test results show that the proposed model can reduce the total intersection delay and the bus passenger waiting time. Altun and Furth found that the optimal performance that used conditional priority under an aggressive schedule can lead to obvious reductions in running time and headway irregularity [26]. A novel method was also proposed by Guler and Gayah that provided priority to buses at signalized intersections using single-lane approaches [27]. The method used addition signals to stop cars on the opposing travel lane so that the bus could use the travel lane in the opposite direction. The results showed that the presignal strategy could reduce the bus and the total passenger delays. The genetic algorithm was used to optimize four basic signal timing parameters and transit priority settings. The results showed that the genetic algorithm elicited a good performance in the optimization of the transit priority setting [28]. Ma et al. assumed that the bus speed is available to adjust in real-time [29]. A set of integrated operational rules which integrated operation of signal timings and bus speed were developed to provide priority to bus at isolated intersections. The experimental analyses show that the proposed integrated operational rules perform better than the priority strategies which do not adjust the bus speed.

Most of the existing transit priority studies have been conducted in low-saturation conditions. It was argued that the intersection under near-saturation conditions is difficult to adopt transit signal priority, owing to the detrimental effects on the no-priority vehicles, which will lead to long vehicular queue dissipation times and long queue lengths [30]. Accordingly, serious traffic jam will be easily caused by assigning transit signal priority. However, from the public transit point of view, it is more meaningful to adopt transit signal priority when the intersection saturation is high. It can improve the level of service of public transit systems which would encourage more travelers to choose the transit mode for their travels.

To remedy this deficiency, this study aims to create a signal control model for transit priority at intersections with high degree of saturation. A passenger-delay-based optimization method is used, in which both the performance of buses and private vehicles are considered. The rest of this study is organized as follows. In Section 2, a green extension model is presented for signal priority on buses that minimizes the total passenger delay at intersections with increased saturations. In Section 3, a case is presented, whereby the signal control model is tested in a near-saturated intersection in Shanghai, China. In Section 4, the sensitivity analysis of the proposed model is presented. In Section 5, conclusions are outlined.

\section{Methodology}

2.1. Basic Idea. To reduce bus delays, especially when these arrive at the end of the green traffic light periods, the green 


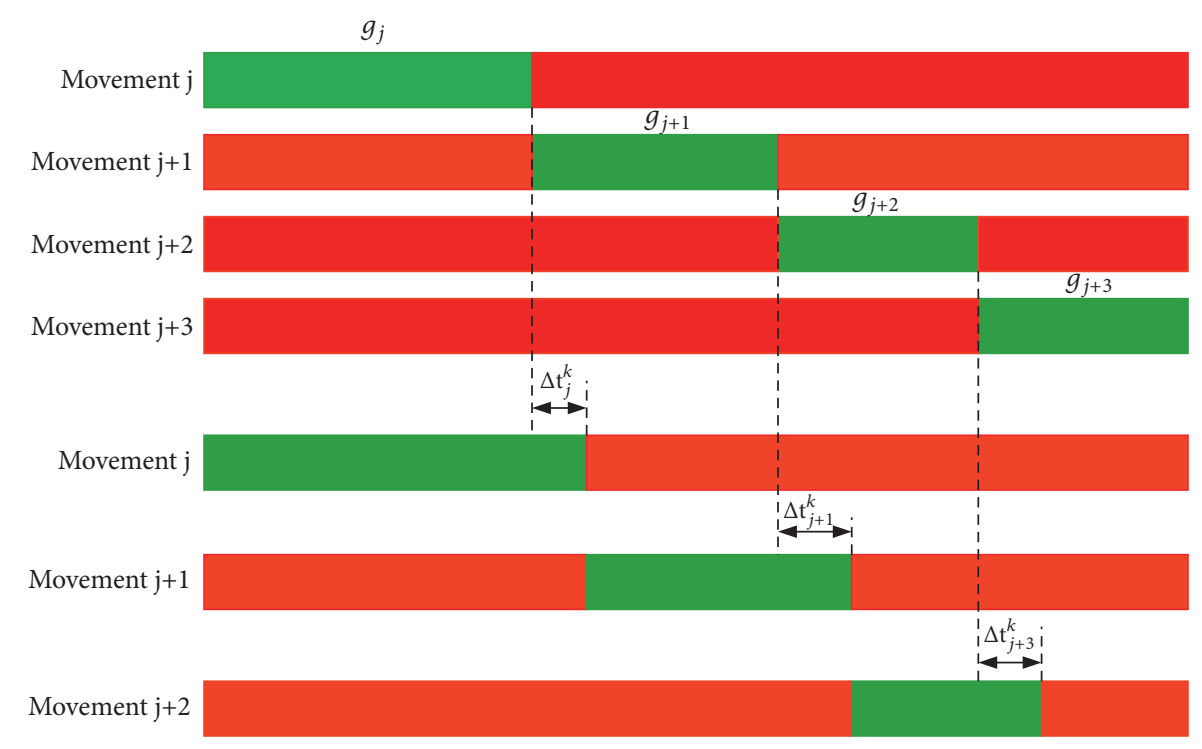

Movement $\mathrm{j}+3$

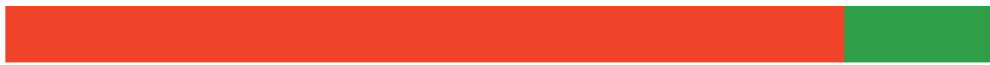

FIGURE 1: Illustration of the green extension strategy.

extension strategy is a common priority strategy that has used in numerous optimization models. Owing to the increase in the time period the green light lasts for buses, the corresponding periods for which the green light is on for other conflicting phases should be shortened. Therefore, the green light period for other phases should be resplit. Correspondingly, three commonly used ways to achieve this include the distribution of all the increased red-light times to a certain phase [31,32], distribution of the increased red-light period to subsequent phases according to the phase splits [33], and the resplitting of the green light period according to the degree of saturation. However, these red-light time distribution strategies may not lead to optimal signal timing results. This study proposed a signal timing optimization model for transit priority at intersections with high degree of saturation to minimize the total intersection passenger delay. As shown in Figure 1, the green light period of movement $j$, which has the same direction as that of the bus, is extended to $g_{j}+\Delta t_{j}^{k}$. The delay of movement $j+1$ will increase owing to the green extension $\Delta \mathrm{t}_{j}^{k}$. To decrease the delay of movement $j+1$, the green light period of movement $j+1$ will be extended to $g_{j+1}-\Delta \mathrm{t}_{j}^{k}+\Delta \mathrm{t}_{j+1}^{k}$, and the same strategy will be adopted in the following movements.

The proposed model aims to minimize the total passenger delay at the intersection by changing the lengths of the green light periods for each movement $\mathrm{j}$. Transit vehicles are assumed to travel on exclusive transit lanes. The dualring, eight-phase structure is considered herein, because it is universally applied in the traffic control industry [34]. The advantage of the dual-ring concept is that it allows the first (usually left-turn) phases in Rings 1 and 2 to terminate independently after their respective demands have been satisfied, which can enhance the flexibility of the signal timing [34]. A four-legged intersection with eight movements is shown in Figure 2(a), and each ring in the controller contains four phases, as depicted in Figure 2(b).

The model will be established in three steps: (1) calculation of the benefits (decreased delay) for private vehicles with the same movements as those for buses, and negative effects (increased delay) for other private vehicles when the green light extension strategy is adopted, (2) establishment of a minimized optimization model that considers the total delay at the intersection, and (3) use of enumeration methods to solve the model. For an easier understanding, a summary of the model notations is presented in Table 1.

2.2. Passenger Delay Increment Calculation. In this study, the total passenger delay increment was used as the indicator and comprised two parts: (1) the passenger delay increment of private vehicles at the intersection and (2) the passenger delay increment of buses at the intersection. The total passenger delay increment was defined as the delay in instances when the bus priority strategy was adopted at intersections minus the delay in instances when no bus priority was allocated at intersections. Therefore, the value of the total passenger delay increment should be negative when the transit signal priority strategy was adopted. Since the proposed model was expected to be used at near-saturated intersections, the application of the transit signal priority may lead to the oversaturation of other traffic movements. For a fair comparison, the analysis included all the cycles until the queue lengths of all the movements were restored to their original conditions. According to the vehicular queue dissipation time, three scenarios are considered herein: (1) scenario 1 , the queue length of movement $j$ can be restored to zero before the end of the green light period 


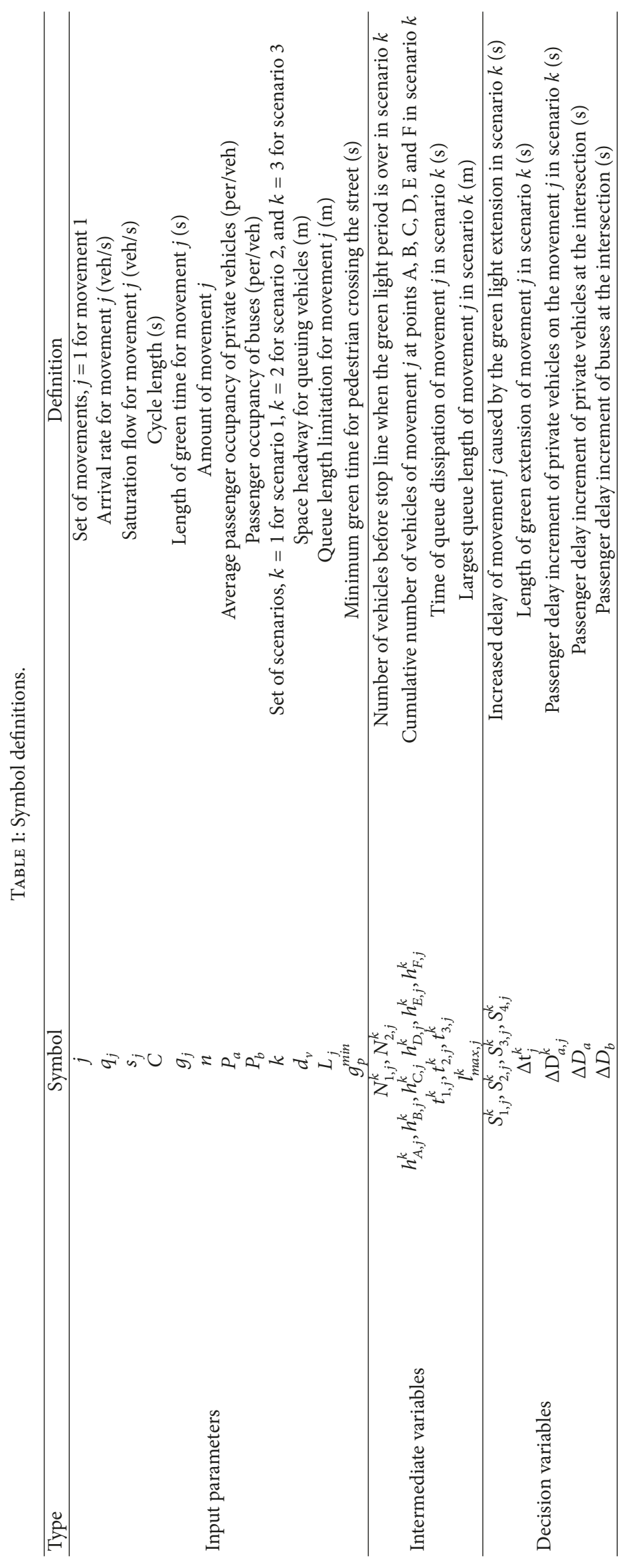




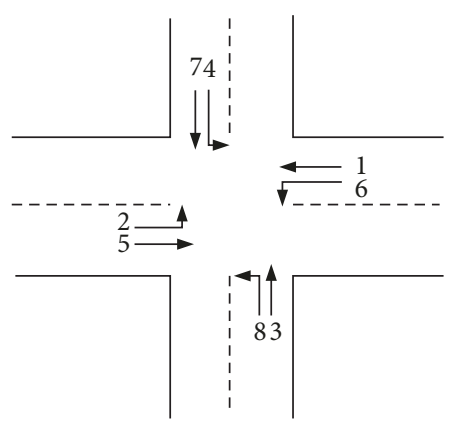

(a) Intersection layout showing the associated phases

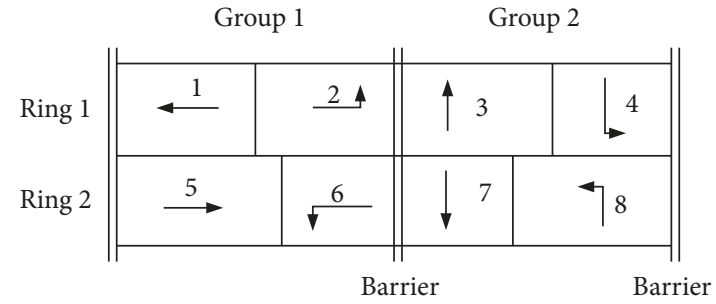

(b) Dual-ring phase structure

FIGURE 2: Phase plan.

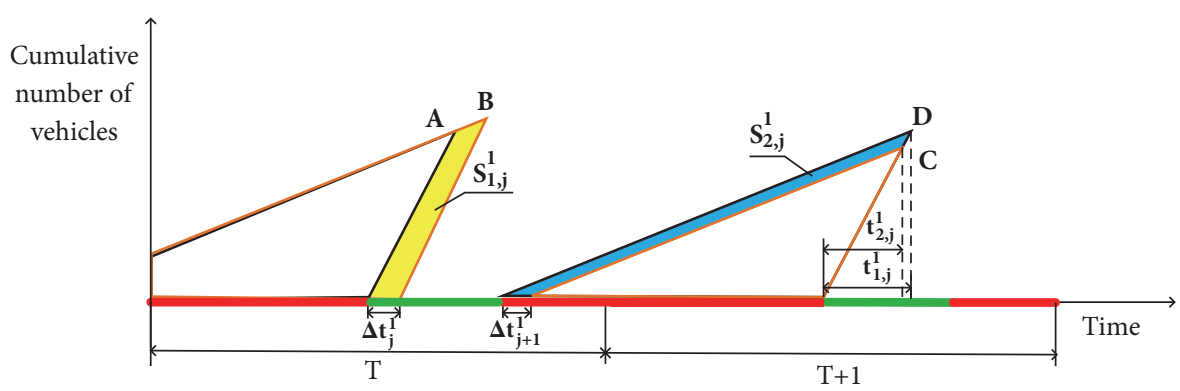

FIGURE 3: Calculation of vehicular delay for movement $\mathrm{j}$ in scenario 1.

that can serve $j$ in the first signal cycle, as shown in Figure 3; (2) scenario 2 , the queue length of movement $j$ can be restored to zero before the end of the green light period that can serve $j$ in the second signal cycle, as shown in Figure 4; (3) scenario 3 , the queue length of movement $j$ can be restored to zero before the end of the green light period that can serve $j$ in the third signal cycle, as shown in Figure 5. When more than three cycles are required for the queue length of movement $j$ to be restored to zero, the total passenger delay increment will be greater than zero. These cases are beyond the scope of the current analyses and are not discussed.

\subsubsection{Passenger Delay Increment of Private Vehicles}

(1) Scenario 1. In scenario 1, the queue length of movement $j$ can be restored to zero before the end of the green light period that can serve $j$ in the first cycle. Figure 3 shows the cumulative number of vehicles present at an intersection for cycle $\mathrm{T}$ and cycle $\mathrm{T}+1$. Without loss of generality, the beginning of the phase that adopts the bus signal priority is set to be the beginning of the signal cycle. The area of the yellow part is the delay increment caused by the extension of the green light, $S_{1, j}^{1}$. The area of blue part is the delay decrement caused by the extension of the green light, $S_{2, j}^{1}$. Then, the passenger delay increment of private vehicles on the movement $j$ in scenario 1 can be calculated by the following:

$$
\Delta D_{a, j}^{1}=P_{a} \cdot\left(S_{1, j}^{1}-S_{2, j}^{1}\right)
$$

Equation (1) is subject to the constraint:

$$
q_{j} \cdot\left(C+\Delta t_{j+1}^{1}\right)-\left(g_{j}-\Delta t_{j}^{1}+\Delta t_{j+1}^{1}\right) \cdot s_{j} \leq 0
$$

Variables in (1), $S_{1, j}^{1}$ and $S_{2, j}^{1}$, can be calculated by (3) and (4), respectively. Intermediate variables, $h_{A, j}^{1}, h_{B, j}^{1} h_{C, j}^{1}$, and $h_{D, j}^{1}$, are the cumulative number of vehicles of movement $j$ at points A-D, which can be calculated by (5)-(8), respectively. Intermediate variables, $t_{1, j}^{1}$ and $t_{2, j}^{1}$, are the time of queue dissipation of movement $j$, which can be calculated by (9) and (10), respectively. Intermediate variable, $l_{\text {max }, j}^{1}$, is the largest queue length of movement $j$ in scenario 1 , which can be calculated by (11).

$$
\begin{aligned}
& S_{1, j}^{1}=\frac{1}{2}\left(C-g_{j}+\Delta t_{j}^{1}\right) h_{B, j}^{1}-\frac{1}{2}\left(C-g_{j}\right) h_{A, j}^{1} \\
& S_{2, j}^{1}=\frac{1}{2}\left(C-g_{j}\right) h_{D, j}^{1}-\frac{1}{2}\left(C-g_{j}-\Delta t_{j+1}^{1}\right) h_{C, j}^{1} \\
& h_{A, j}^{1}=\frac{s_{j} q_{j}\left(C-g_{j}\right)}{s_{j}-q_{j}} \\
& h_{B, j}^{1}=\frac{s_{j} q_{j}\left(C-g_{j}+\Delta t_{j}^{1}\right)}{s_{j}-q_{j}} \\
& h_{C, j}^{1}=\frac{s_{j} q_{j}\left(C-g_{j}-\Delta t_{j+1}^{1}\right)}{s_{j}-q_{j}}
\end{aligned}
$$




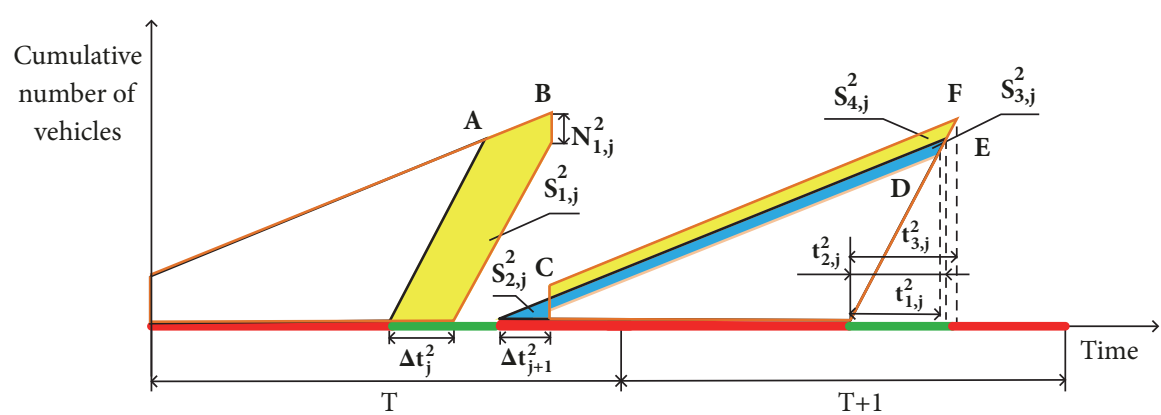

FIGURE 4: Calculation of vehicular delay for movement $\mathrm{j}$ in scenario 2.

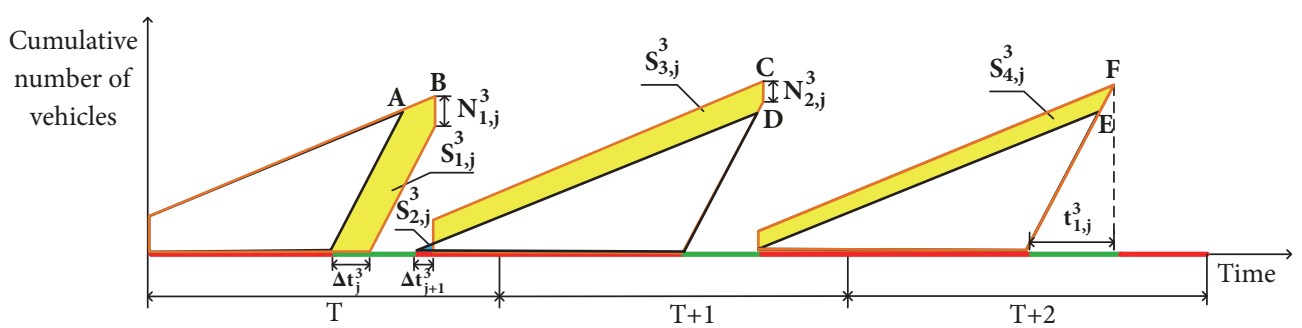

Figure 5: Calculation of vehicular delay for movement $\mathrm{j}$ in scenario 3.

$$
\begin{aligned}
h_{D, j}^{1} & =h_{A, j}^{1} \\
t_{1, j}^{1} & =\frac{q_{j} \cdot\left(C-g_{j}\right)}{s_{j}-q_{j}} \\
t_{2, j}^{1} & =\frac{q_{j} \cdot\left(C-g_{j}-\Delta t_{j+1}^{1}\right)}{s_{j}-q_{j}} \\
l_{\text {max }, j}^{1} & =h_{B, j}^{1} d_{v}=\frac{s_{j} d_{v} q_{j}\left(C-g_{j}+\Delta t_{j}^{1}\right)}{s_{j}-q_{j}}
\end{aligned}
$$

(2) Scenario 2. In scenario 2, the queue length of movement $j$ can be restored to zero before the end of the green light period that can serve $j$ in the second cycle. Figure 4 shows the cumulative number of vehicles present at an intersection for cycle $\mathrm{T}$ and cycle $\mathrm{T}+1$. The areas of the yellow parts, $S_{1, j}^{2}$ and $S_{4, j}^{2}$, are the delay increments caused by the extensions of the green light periods $\Delta \mathrm{t}_{j}^{2}$ and $\Delta \mathrm{t}_{j+1}^{2}$, respectively. The areas of the blue parts, $S_{2, j}^{2}$ and $S_{3, j}^{2}$, are the delay decrement caused by the extension of the green light $\Delta \mathrm{t}_{j+1}^{2}$. Then, the passenger delay increment of private vehicles on the movement $j$ in scenario 2 can be calculated by (12). In (12), the condition $N_{1, j}^{2} \geq \Delta t_{j+1}^{2} q_{j}$ implies that the queue length $N_{1, j}^{2}$ is larger than or equal to the queue length when the movement $j$ does not adopt the extension of the green light period $\Delta t_{j+1}^{2}$. The condition $N_{1, j}^{2}<\Delta t_{j+1}^{2} q_{j}$ implies that the queue length $N_{1, j}^{2}$ is less than the queue length in the instance when the movement $j$ does not adopt the extension of the green light period $\Delta t_{j+1}^{2}$.

$$
\Delta D_{a, j}^{2}= \begin{cases}P_{a}\left(S_{1, j}^{2}-S_{2, j}^{2}+S_{4, j}^{2}\right), & N_{1, j}^{2} \geq \Delta t_{j+1}^{2} q_{j} \\ P_{a}\left(S_{1, j}^{2}-S_{2, j}^{2}-S_{3, j}^{2}\right), & N_{1, j}^{2}<\Delta t_{j+1}^{2} q_{j}\end{cases}
$$

Equation (12) is subject to the restrictions:

$$
\begin{aligned}
q_{j}\left(C+\Delta t_{j+1}^{2}\right)-s_{j}\left(g_{j}-\Delta t_{j}^{2}+\Delta t_{j+1}^{2}\right) & \geq 0 \\
q_{j}(2 C)-s_{j}\left(2 g_{j}-\Delta t_{j}^{2}+\Delta t_{j+1}^{2}\right) & \leq 0
\end{aligned}
$$

Variables in (12), $S_{1, j}^{2}, S_{2, j}^{2}, S_{3, j}^{2}$, and $S_{4, j}^{2}$, can be calculated by (15)-(18), respectively. The intermediate variable $N_{1, j}^{2}$ denotes the number of vehicles when the green light period is completed, which can be calculated by (19). The intermediate variables $h_{A, j}^{2}, h_{B, j}^{2}, h_{C, j}^{2}, h_{D, j}^{2}, h_{E, j}^{2}$, and $h_{F, j}^{2}$ are the cumulative numbers of vehicles for movement $j$ at points $\mathrm{A}-\mathrm{F}$, which can be calculated by (20)-(25), respectively. The intermediate variables $t_{1, j}^{2}, t_{2, j}^{2}$, and $t_{3, j}^{2}$ are the queue dissipation time for movement $j$, which can be calculated by (26)-(28), respectively. The intermediate variable $l_{\text {max,j }}^{2}$ is the largest queue length of movement $j$, which can be calculated by (29).

$$
\begin{aligned}
S_{1, j}^{2}= & \frac{1}{2}\left(C+\Delta t_{j+1}^{2}\right) h_{B, j}^{2}-\frac{1}{2}\left(C-g_{j}\right) h_{A, j}^{2} \\
& -\frac{1}{2}\left(g_{j}-\Delta t_{j}^{2}+\Delta t_{j+1}^{2}\right)\left(h_{B, j}^{2}-N_{1, j}^{2}\right) \\
S_{2, j}^{2}= & \frac{1}{2} \Delta \mathrm{t}_{j+1}^{2} \Delta \mathrm{t}_{j+1}^{2} q_{j} \\
S_{3, j}^{2}= & \frac{1}{2}\left(N_{1, j}^{2}+h_{D, j}^{2}\right)\left(C-g_{j}-\Delta t_{j+1}^{2}+t_{j, 1}^{2}\right) \\
& -\frac{1}{2} t_{1, j}^{2} h_{D, j}^{2} \\
& -\frac{1}{2}\left(\Delta t_{j+1}^{2} q_{j}+h_{E, j}^{2}\right)\left(C-g_{j}-\Delta t_{j+1}^{2}+t_{2, j}^{2}\right) \\
& +\frac{1}{2} t_{2, j}^{2} h_{E, j}^{2}
\end{aligned}
$$




$$
\begin{aligned}
& S_{4, j}^{2}= \frac{1}{2}\left(N+h_{F, j}^{2}\right)\left(C-g_{j}-\Delta t_{j+1}^{2}+t_{3, j}^{2}\right) \\
&-\frac{1}{2} t_{3, j}^{2} h_{F, j}^{2} \\
&-\frac{1}{2}\left(\Delta t_{j+1}^{2} q_{j}+h_{E, j}^{2}\right)\left(C-g_{j}-\Delta t_{j+1}^{2}+t_{2, j}^{2}\right) \\
&+\frac{1}{2} t_{2, j}^{2} h_{E, j}^{2} \\
& N_{1, j}^{2}= q_{j} \cdot\left(C+\Delta t_{j+1}^{2}\right)-s_{j}\left(g_{j}-\Delta t_{j}^{2}+\Delta t_{j+1}^{2}\right) \\
& h_{A, j}^{2}= \frac{q_{j}\left(C-g_{j}\right)}{s_{j}-q_{j}} s_{j} \\
& h_{B, j}^{2}= q_{j}\left(C+\Delta t_{j+1}^{2}\right) \\
& h_{C, j}^{2}= N_{1, j}^{2} \\
& h_{D, j}^{2}= s_{j} t_{1, j}^{2} \\
& h_{E, j}^{2}= s_{j} t_{2, j}^{2} \\
& h_{F, j}^{2}= s_{j} t_{3, j}^{2} \\
& t_{1, j}^{2}= \frac{N_{j, 1}^{2}+q_{j} \cdot\left(C-g_{j}-\Delta t_{j+1}^{2}\right)}{s_{j}-q_{j}} \\
& t_{2, j}^{2}= \frac{q_{j}\left(C-g_{j}\right)}{s_{j}-q_{j}} \\
& t_{m a x, j}^{2}= \frac{N_{1, j}^{2}+q_{j} \cdot\left(C-g_{j}-\Delta t_{j+1}^{2}\right)}{s_{j}-q_{j}} \\
& h_{v}=q_{j}\left(C+\Delta t_{j+1}^{2}\right) d_{v} \\
& d^{2}
\end{aligned}
$$

(3) Scenario 3. In scenario 3, the queue length of group $j$ can be restored to zero before the end of the green time period that can serve $j$ in the third cycle. Figure 5 shows the cumulative number of vehicles present at an intersection for cycles $\mathrm{T}, \mathrm{T}+1$, and $\mathrm{T}+2$. The area of the yellow part, $S_{1, j}^{3}$, $S_{3, j}^{3}, S_{4, j}^{3}$, is the delay increments caused by the extensions of the periods when the green light was on. The area of the blue part, $S_{2, j}^{3}$, is the delay decrement caused by the extension of the green light period. Then, the passenger delay increment of private vehicles on the movement $j$ in scenario 3 can be calculated by (30).

$$
\Delta D_{a, j}^{3}=P_{a}\left(S_{1, j}^{3}-S_{2, j}^{3}+S_{3, j}^{3}+S_{4, j}^{3}\right)
$$

Equation (30) is subject to the restrictions:

$$
\begin{aligned}
& q_{j}(2 C)-\left(2 g_{j}-\Delta t_{j}^{3}+\Delta t_{j+1}^{3}\right) s_{j} \geq 0 \\
& q_{j}(3 C)-\left(3 g_{j}-\Delta t_{j}^{3}+\Delta t_{j+1}^{3}\right) s_{j} \leq 0
\end{aligned}
$$

Variables in (12), $S_{1, j}^{3}, S_{2, j}^{3}, S_{3, j}^{3}$, and $S_{4, j}^{3}$, can be calculated by (33)-(36), respectively. Intermediate variables $N_{1, j}^{3}$ and $N_{2, j}^{3}$ are the number of vehicles when the time period of the green light is completed, which can be calculated by (37) and (38), respectively. The intermediate variables $h_{A, j}^{3}, h_{B, j}^{3}, h_{C, j}^{3}$, $h_{D, j}^{3}, h_{E, j}^{3}$, and $h_{F, j}^{3}$ are the cumulative number of vehicles of movement $j$ at points $\mathrm{A}-\mathrm{F}$, which can be calculated by (39) and (44), respectively. The intermediate variable $t_{1, j}^{3}$ is the time of queue dissipation of movement $j$, which can be calculated by (45). The intermediate variable $l_{\text {max }, j}^{3}$ is the largest queue length of movement $j$, which can be calculated by (46).

$$
\begin{aligned}
S_{1, j}^{3}= & \frac{1}{2}\left(C+\Delta t_{j+1}^{3}\right) h_{B, j}^{3}-\frac{1}{2}\left(C-g_{j}\right) h_{A, j}^{3} \\
& -\frac{1}{2}\left(g_{j}-\Delta t_{j}^{3}+\Delta t_{j+1}^{3}\right)\left(h_{B, j}^{3}-N_{1, j}^{3}\right) \\
S_{2, j}^{3}= & \frac{1}{2} \Delta \mathrm{t}_{j+1}^{3} \Delta \mathrm{t}_{j+1}^{3} q_{j} \\
S_{3, j}^{3}= & \frac{1}{2}\left(N_{1, j}^{3}+h_{C, j}^{3}\right)\left(C-\Delta t_{j+1}^{3}\right) \\
& -\left[\frac{1}{2}\left(C-g_{j}\right) h_{D, j}^{3}-S_{2}\right] \\
& -\frac{1}{2} g_{j}\left(h_{D, j}^{3}-N_{2, j}^{3}\right) \\
S_{4, j}^{3}= & \frac{1}{2}\left(N_{2, j}^{3}+h_{F, j}^{3}\right)\left(\mathrm{C}-g_{j}+t_{1, j}^{3}\right) \\
& -\frac{1}{2}\left(\mathrm{C}-g_{j}\right) h_{E, j}^{3}-\frac{1}{2} t_{1, j}^{3} h_{F, j}^{3} \\
N_{1, j}^{3}= & q_{j}\left(C+\Delta t_{j+1}^{3}\right)-s_{j}\left(g_{j}-\Delta t_{j}^{3}+\Delta t_{j+1}^{3}\right) \\
N_{2, j}^{3}= & N_{1, j}^{3}+q_{j}\left(C-\Delta t_{j+1}^{3}\right)-s_{j} g_{j} \\
t_{1, j}^{3}= & \frac{N_{j, 2}^{3}+q_{j}\left(C-g_{j}\right)}{s_{j}-q_{j}} \\
h_{A, j}^{3}= & \frac{s_{j} q_{j}\left(C-g_{j}\right)}{s_{j}-q_{j}} \\
h_{B, j}^{3}= & q_{j}\left(C+\Delta t_{j+1}^{3}\right) \\
h_{C, j}^{3}= & N_{1, j}^{3}+q_{j}\left(C-\Delta t_{j+1}^{3}\right) \\
h_{D, j}^{3}= & \frac{s_{j} q_{j}\left(C-g_{j}\right)}{s_{j}-q_{j}} \\
h_{E, j}^{3}= & \frac{s_{j} q_{j}\left(\mathrm{C}-g_{j}\right)}{s_{j}-q_{j}} \\
N_{j, 2}^{3}+q_{j} \cdot\left(C-g_{j}\right) & s_{j}-q_{j} \\
h_{1, j} &
\end{aligned}
$$




$$
l_{\text {max }, j}^{3}=h_{C, j}^{3} d_{v}=\left[N_{1, j}^{3}+q_{j}\left(C-\Delta t_{j+1}^{3}\right)\right] d_{v}
$$

In summary, the passenger delay increment of buses at the intersection on movement $j$ in scenarios 1-3 can be expressed as (47). For the scenario 1, 2, and 3, (2), (13)-(14), and (31)-(32) should be satisfied, respectively. The passenger delay increment of private vehicles, $\Delta D_{a}$, equals the sum of the passenger delay increment for private vehicles of all movements, as shown in (48).

$$
\begin{aligned}
\Delta D_{j, a}= & \begin{cases}P_{a} \cdot\left(S_{1, j}^{1}-S_{2, j}^{1}\right), & \text { scenario 1 } \\
P_{a} \cdot\left(S_{1, j}^{2}-S_{2, j}^{2}+S_{3, j}^{2}+S_{4, j}^{2}\right), & \text { scenario 2 } \\
P_{a} \cdot\left(S_{1, j}^{3}-S_{2, j}^{3}+S_{3, j}^{3}+S_{4, j}^{3}\right), & \text { scenario 3 }\end{cases} \\
\Delta D_{a}=\sum_{j=1}^{n} \Delta D_{j, a} \# &
\end{aligned}
$$

2.2.2. Passenger Delay Increment of Buses. The passenger delay increment of buses, $\Delta D_{b}$, is the negative value of the length of the remaining red light of movement 1 , as shown in the following:

$$
\Delta D_{b}=-P_{b} \cdot\left(C-g_{1}-\Delta t_{1}^{k}\right)
$$

2.2.3. Validation of the Delay Model. The delay increment calculation should be validated through the microscopic simulation package VISSIM. The basic idea of delay increment calculation validation is as follows: as shown in (48), the passenger delay increment of private vehicles, $\Delta D_{a}$, equals the sum of the passenger delay increment for private vehicles of all movements, so the delay calculation of each movement is calculated by the same equation (47). In other words, the validation of the passenger delay increment calculation is actually to validate (47).

In the following validation process, a test movement is selected, the basic parameters of the test movement and the green light extension time are input, and, after the simulation, the total vehicular delay of the movement is output. Because the correctness of the delay increment calculation under different scenarios needs to be tested, different green light extension times are input, which can result in shorter green times assigned to the vehicle and the queue length needing multiple cycles to return to zero. Finally, the total vehicular delay of the movement under the three scenarios is recorded and compared with the calculated values of the model.

The value of the input parameters are as follows: the cycle length is $100 \mathrm{~s}$, the green time of the test lane is $25 \mathrm{~s}$, the arrival rate and the saturation rate of the test lane are 360 and $1800 \mathrm{veh} / \mathrm{h}$ respectively, and the green extension time will be from 1 to 10 . If the green extension time is $1,2,3$, the queue length of test movement will be restored to zero in first cycle, so the delay increment calculation in scenario 1 will be validated. If the green extension time is $4,5,6$, the queue length of test movement will be restored to zero in second cycle, so the delay increment calculation in scenario 2 will be validated. If the green extension time is $7,8,9,10$, the queue length of test movement will be restored to zero in

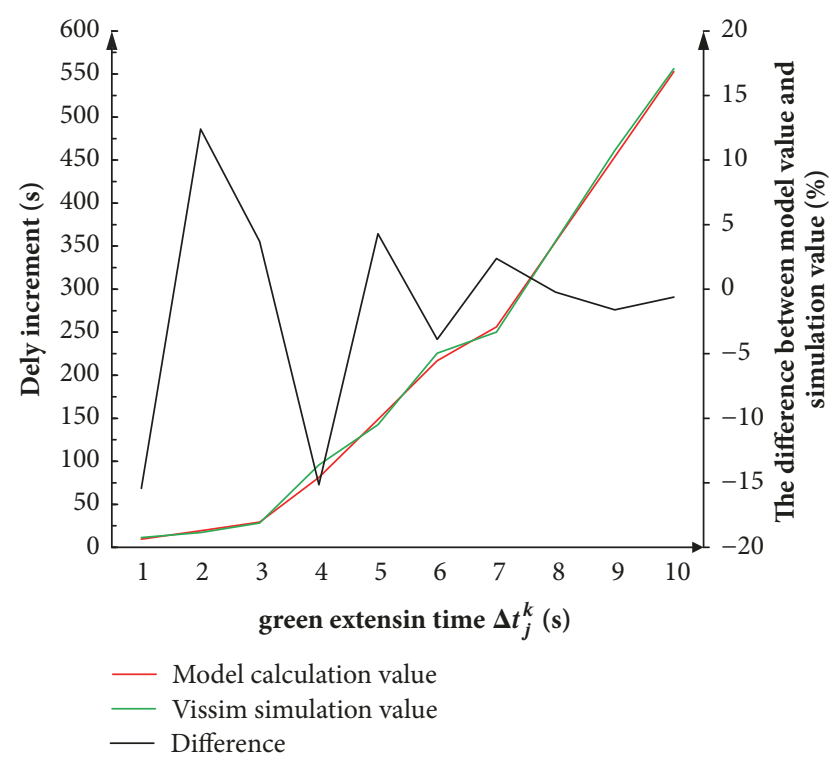

FIGURE 6: Comparison between the model calculation value and the simulation value.

third cycle, so the delay increment calculation in scenario 3 will be validated. The simulation time of VISSIM is set to 4000 s. The delay increment calculation validation results are presented in Figure 6. As depicted in Figure 6, the delay increment calculation value is close to the simulation value, and the maximum difference does not exceed $16 \%$, the delay increment calculation is proved to be valid.

\subsection{Optimization Model}

2.3.1. Objective. The transit signal priority strategy is to improve the operational efficiency. Minimizing the total passenger delay was selected as the optimization objective, which is equivalent to minimizing the total passenger delay increment caused by the transit signal priority strategy, as shown in (50), in which the passenger delay increment of private vehicles and buses can be calculated by (48) and (49), respectively.

$$
\min \Delta D_{a}+\Delta D_{b}
$$

2.3.2. Constraints. To avoid vehicle queue spillovers, the queue length cannot be greater than the maximum queue length limitation, $L_{\max }$, as shown in the following:

$$
l_{\text {max }, j}^{k} \leq L_{\text {max }}, \quad \forall k=1,2,3
$$

The green light extension for movement $j+1$ is less than or equal to the green light extension for movement $j$, as shown in (52). For $\Delta \mathrm{t}_{4}^{k}$ and $\Delta \mathrm{t}_{8}^{k}$, they are at the end-periods of the ring and cannot extend the green light period to the next movement. Therefore, they are equal to zero, as shown in (53). Moreover, in the dual-ring, all phases in one group must 
TABLE 2: Traffic parameters of the tested intersection.

\begin{tabular}{lccc}
\hline Intersection approach & Movements & Traffic volume $(\mathrm{veh} / \mathrm{h})$ & Saturation flow rate $(\mathrm{veh} / \mathrm{h})$ \\
\hline \multirow{3}{*}{ East } & Left turn & 728 & 3100 \\
& Through & 1108 & 5800 \\
& Right turn & 588 & 1550 \\
\multirow{3}{*}{ West } & Left turn & 294 & 1400 \\
& Through & 1368 & 8000 \\
& Right turn & 696 & 1550 \\
North & Left turn & 864 & 3200 \\
& Through & 540 & 2000 \\
& Right turn & 104 & 775 \\
South & Left turn & 124 & 700 \\
& Through & 524 & 3400 \\
\hline
\end{tabular}

TABLE 3: Number of cycles required for recovery.

\begin{tabular}{lccccccc}
\hline Cycles for recovery & M1 & M2 & M3 & M4 & M5 & M6 & M7 \\
\hline Proposed method & 1 & 2 & 1 & 1 & 1 & 1 & 1 \\
Traditional method A & 1 & 1 & 2 & 3 & 1 & 1 & 2 \\
Traditional method B & 1 & 1 & 2 & 3 & 1 & 1 & 2 \\
\hline
\end{tabular}

Note: $\mathrm{M}=$ movement.

be terminated before the beginning of any of the phases of the next group. Thus, $\Delta \mathrm{t}_{2}^{k}$ should be equal to $\Delta \mathrm{t}_{6}^{k}$, as shown in (54).

$$
\begin{aligned}
0 & \leq \Delta t_{j+1}^{k} \leq \Delta t_{j}^{k}, \quad \forall j=1,2,3,6,7 ; k=1,2,3 \\
\Delta t_{4}^{k} & =\Delta t_{8}^{k}=0 \\
\Delta t_{2}^{k} & =\Delta t_{6}^{k}
\end{aligned}
$$

The green time under the dual-ring phase plan should meet the constraint (55) due to the setting of the barriers shown in Figure 2(b). Moreover, the minimum green time period should be larger than or equal to the minimum green light period needed for the pedestrians to cross the street, as shown in (56).

$$
\begin{gathered}
g_{j}+g_{j+1}+\Delta t_{j+1}^{k}=g_{j+4}+g_{j+5}+\Delta t_{j+5}^{k}, \\
\forall j=1,3 . k=1,2,3 \\
g_{j}-\Delta t_{j}^{k}+\Delta t_{j+1}^{k} \geq g_{p}^{\min }, \quad \forall k=1,2,3
\end{gathered}
$$

2.3.3. Solution. The proposed model was coded in MATLAB and tested on an Intel i5, 2.5 GHz processor and 4.0 GB RAM, running Windows, and was solved using an enumeration method. The program running interface is presented in Figure 7.

\section{Case Study}

The proposed model is applied to a real-world intersection, namely, the intersection of the Huashan and Middle Yan'an roads located in Shanghai, China. The intersection's layout is presented in Figure 8(a). As shown in this figure, the main through movement is via Yan'an road. The exclusive bus lane is set on Middle Yan'an road. The traffic volumes during the morning peak hours (8 to 9 a.m.) were obtained based on a field survey. The real data acquired from the Huashan and Middle Yan'an roads are presented in Table 2. The intersection is operated on a fixed four-phase cycle with a cycle length of $190 \mathrm{~s}$, as presented in Figure 8(b). The saturation of the intersection is $85.2 \%$. The average passenger occupancy of private vehicles is $1.5 \mathrm{per} / \mathrm{veh}$; the passenger occupancy of buses is $20 \mathrm{per} / \mathrm{veh}$.

Now, there is an application of the bus priority. The required bus priority time is $18 \mathrm{~s}$. In order to better reflect the advantages of the proposed model, the basic timing plan will be adjusted, the existing timing plan will be optimized according to actual traffic flow. In this way, the problem of unreliable model results due to the irrationality of the basic signal timing can be solved. The adjusted signal timing is presented in Figure 9. The proposed model is used to optimize the adjusted signal timing. The running time in MATLAB is $1.72 \mathrm{~s}$. The optimization results include the extension time for the green light period $\Delta \mathrm{t}_{1}^{k}=18 \mathrm{~s}, \Delta \mathrm{t}_{2}^{k}=8 \mathrm{~s}$, $\Delta \mathrm{t}_{4}^{k}=2 \mathrm{~s}, \Delta \mathrm{t}_{6}^{k}=8 \mathrm{~s}, \Delta \mathrm{t}_{7}^{k}=5 \mathrm{~s}$. The optimized signal timing is presented in Figure 10.

The performance of the proposed model is compared with two traditional methods, namely, traditional methods A and $\mathrm{B}$ as shown in Tables $3-5$. The traditional method A is the method which distributes red-light time to the following phase according to the phase splits. The traditional method $B$ is the method which resplits the green light time period according to the degree of saturation.

As Table 3 shows, the queue length of movement $j$ will be restored to zero in two cycles. As Table 4 shows, the 
TABLE 4: Largest queue length.

\begin{tabular}{lcccccccc}
\hline Queue length $(\mathrm{m})$ & M1 & M2 & M3 & M4 & M5 & M6 & M7 & M8 \\
\hline Proposed method & 77.2 & 81.7 & 126.0 & 68.4 & 62.5 & 94.5 & 118.5 & 66.8 \\
Traditional method A & 77.2 & 80.5 & 130.5 & 74.1 & 62.5 & 94.5 & 122.7 & 66.8 \\
Traditional method B & 77.2 & 80.8 & 130.2 & 72.2 & 62.5 & 94.5 & 122.5 & 65.4 \\
\hline
\end{tabular}

Note: $\mathrm{M}=$ movement.

TABLE 5: Comparisons of three methods about delay variation.

\begin{tabular}{lcccc}
\hline Schemes & Index & Total passenger delay $(\mathrm{s})$ & Bus passenger delay (s) & Auto passenger delay $(\mathrm{s})$ \\
\hline No transit signal priority & Mean & 33333.6 & 2640.0 & 30693.6 \\
Proposed method & Mean & 31849.9 & 0 & 31849.9 \\
& variation & $-4.5 \%$ & $-100 \%$ & $3.8 \%$ \\
Traditional method A & Mean & 34291.3 & 0 & 34291.3 \\
& variation & $2.9 \%$ & $-100 \%$ & $11.7 \%$ \\
Traditional method B & Mean & 33498.3 & 0 & 33498.3 \\
& variation & $0.5 \%$ & $-100 \%$ & $9.1 \%$ \\
\hline
\end{tabular}

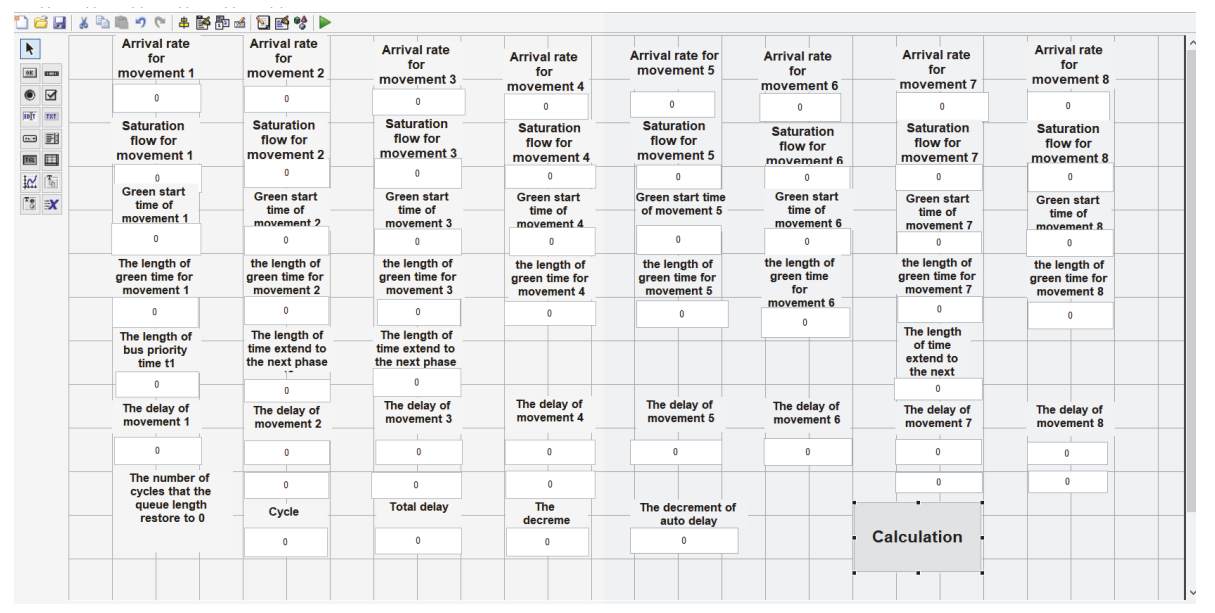

FIgURE 7: Program running interface.

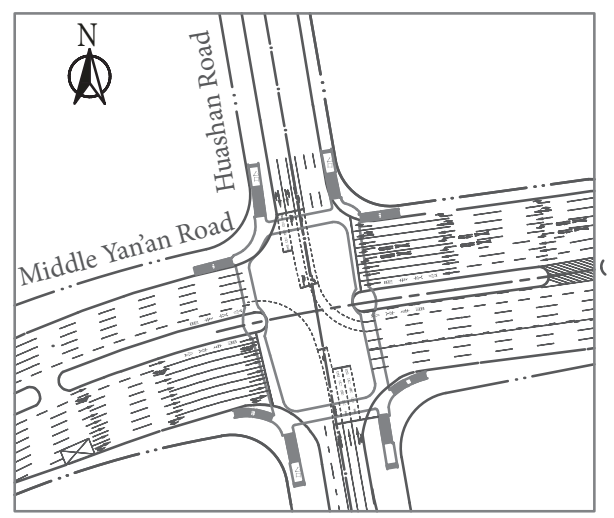

(a) Original layout

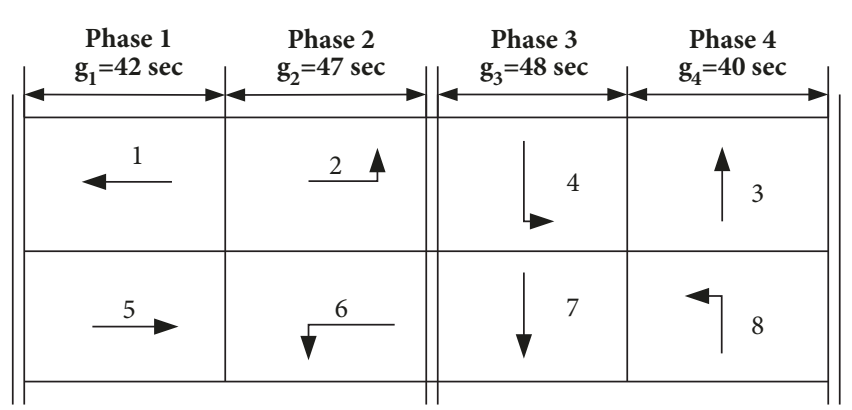

(b) Original signal timing

FIGURE 8: Original layout and signal timing of the tested intersection. 


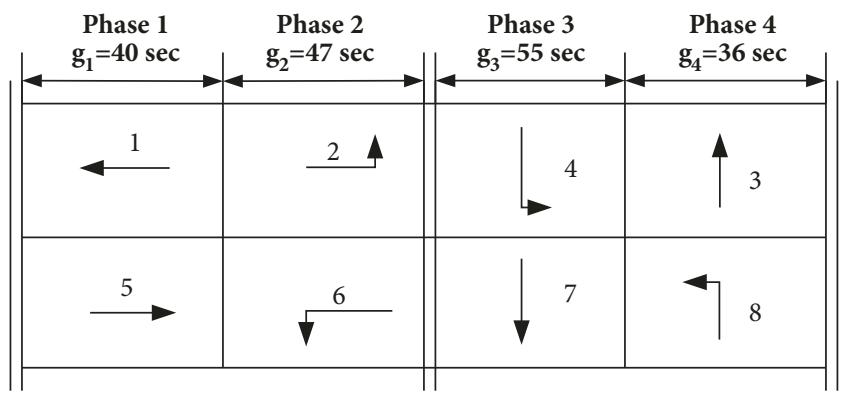

FIGURE 9: Adjusted signal timing.

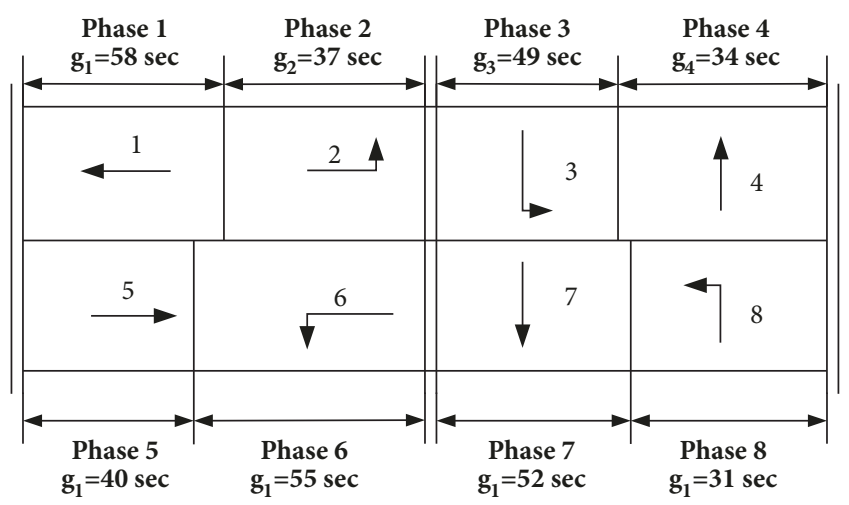

FIGURE 10: Optimized signal timing with transit signal priority.

largest queue length of movement $j$ is less than $150 \mathrm{~m}$, which is less than the road length. Thus, the proposed method is suitable for this intersection. Moreover, the proposed method outperforms the traditional methods in terms of the recovery time and queue length. All the movements can be recovered within 2 cycles for the proposed method, while it requires 3 cycles for recovery. The average and longest queue length for the proposed method is shortened by $1.7 \%$ and $3.4 \%$, respectively, with the comparison of the traditional methods.

Table 5 compares the three methods from the perspective of the delay variation in three signal cycles. As Table 5 shows, the proposed method can reduce the total passenger delay and the percentage of delay decrement by $4.5 \%$. Conversely, the traditional method will increase the total passenger delay. In addition, the percentage of the auto-delay increment is more than $9.1 \%$ after the optimization of the traditional method, which is larger than $3.8 \%$.

\section{Sensitivity Analysis}

A sensitivity analysis was conducted in order to analyze the influence of the intersection's geometry and traffic conditions on the efficiency of the proposed model. The length of the priority time for buses, the intersection saturation, and the number of lanes were selected as the three key parameters for the sensitivity analysis. The value of the input parameters are as follows: in Figures 11, 12, and 14, the cycle length is $80 \mathrm{~s}$, there are four phases and the time of each phase is $20 \mathrm{~s}$, the arrive rate of each movement is the same, which is from 315 to $472 \mathrm{veh} / \mathrm{h}$ depending on the saturation, the saturation rate of each movement is $1800 \mathrm{veh} / \mathrm{h}$, the average passenger occupancy of private vehicles is 1.5 per/veh, the passenger occupancy of buses is $20 \mathrm{per} / \mathrm{veh}$, and the number of lanes is three. In Figure 13, the arrival rate of each movement is 382 $\mathrm{veh} / \mathrm{h}$, and other parameters are the same as above.

In order to avoid the irrationality of the basic signal timing results to unreliable model results, the signal timing and the intersection conditions are set as above described, the signal timing has been set to be optimal. Figure 11 shows the relationship between the delay increment and the three key parameters. Figure 11 is composed of 9 subfigures. The ordinates and abscissa of Figure 11 are priority time and number of lanes, respectively. The ordinates and abscissa of each subfigure are saturation and delay increment, respectively. The nine subfigures of Figure 9 are arranged such that the priority time gradually increases from the bottom to the top, and the number of lanes gradually increases from the left to the right.

From the perspective of each of these subfigures, the vehicular delay increases as the saturation increases; however, the delay of the bus will not change. Additionally, comparison of these subfigures from the bottom to the top indicates that the vehicular delay increases as the priority increases. In contrast, the bus delay decrement becomes smaller. Comparison of the data in the subfigures from the left to the right indicates that the vehicular delay increases as the number of lanes increase, while the bus delay does not change. The reason for which the vehicular delay increases as the number of lanes increases is attributed to the fact that as the number of lanes increases, the number of vehicles will increase if the saturation of the intersection remains constant.

Figure 12 further shows the combination effects of the saturation of intersection and the priority time. As indicated, the total delay will increase as the priority time and the saturation increase. The delay increment increases rapidly and becomes very large as the saturation of intersection becomes larger than 0.9. Similarly, the delay increment will increase rapidly and will become very large as the priority time becomes larger than $10 \mathrm{~s}$.

As Figure 12 shows, the delay increment is sensitive to the priority time. Thus, a better optimization model is needed which is less sensitive to the priority time. As Figure 13 shows, the two traditional methods get the same results, the reason is that the basic signal timing is optimal, the splits and degree of saturation of each phase is the same. Figure 13 compares the proposed model and the traditional model, based on which several inferences can be drawn: (1) the proposed model is less sensitive than the traditional model to the priority time; (2) the difference of the effect of optimization is not obvious between the proposed model and traditional method when the priority time is less than $5 \mathrm{~s}$, and (3) when the priority time is larger than $5 \mathrm{~s}$, the difference of the effects of the optimization between the two methods will increase, as the priority time will increase. When the priority time becomes larger than $9 \mathrm{~s}$, the proposed model is $15 \%$ better than the traditional models A and B.

Figure 14 presents a table listing the recommended values for bus signal priorities, which can provide suggestions for practical applications. As Figure 14 shows, the percentages in this figure are the percentage reductions of the total 


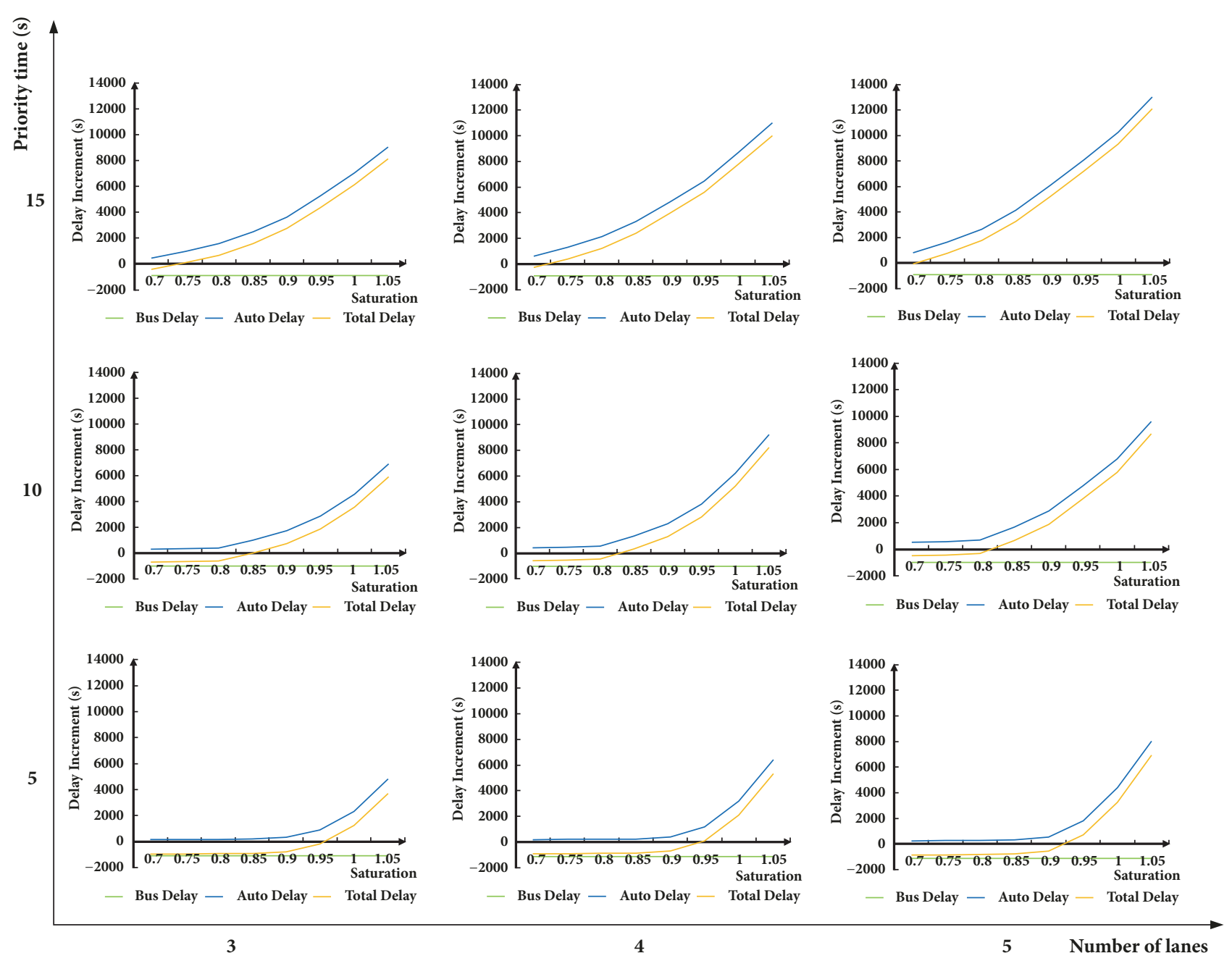

FIGURE 11: Sensitivity plots of the total delay increment to the priority time and the number of lanes.

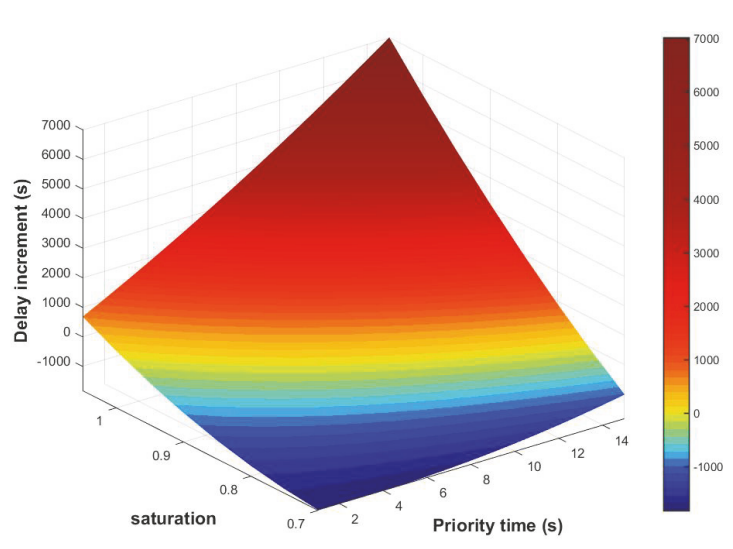

FIGURE 12: Sensitivity of the total delay increment as a function of the priority time and saturation.

passenger delays at the intersection when bus signal priorities are assigned. Figure 14 contains three parts, namely, the green part that is suitable for application of the bus signal priority strategy, the red part that is unsuitable for application of the bus signal priority strategy, and the yellow part that indicates that the percentage reduction of the total delay is larger than zero but less than $10 \%$. In the yellow highlighted part, the effect of the bus signal priority strategy is minor.

\section{Conclusions}

This paper presented a signal control model for assigning bus signal priorities that also minimized the total passenger delay increment at intersections with the near-saturation. The proposed model was tested with simulations at a nearsaturated intersection in Shanghai, China. Several meaningful conclusions can be drawn from the sensitivity analyses:

(1) Bus signal priority can be applied in near-saturated intersections, whereby the length of priority time, the saturation of the intersection, and the number of lanes were the three main factors that influenced whether the intersections should have adopted the bus signal priority strategy.

(2) The method on resplitting the green light time period affected the benefits of the bus signal priority considerably. 


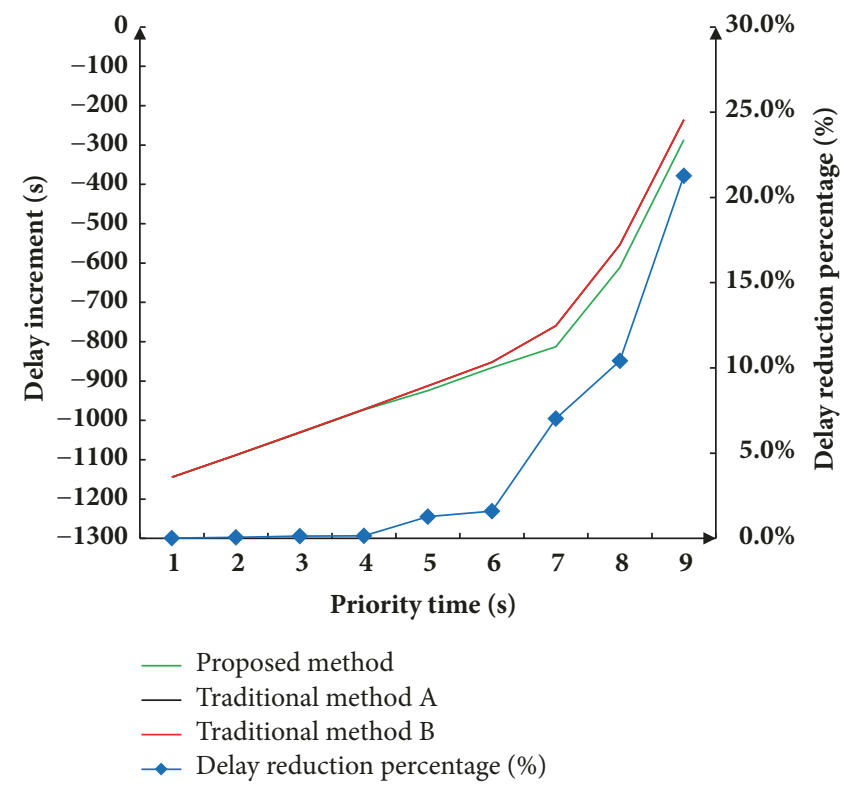

FIGURE 13: Comparison of the proposed model to the traditional model.

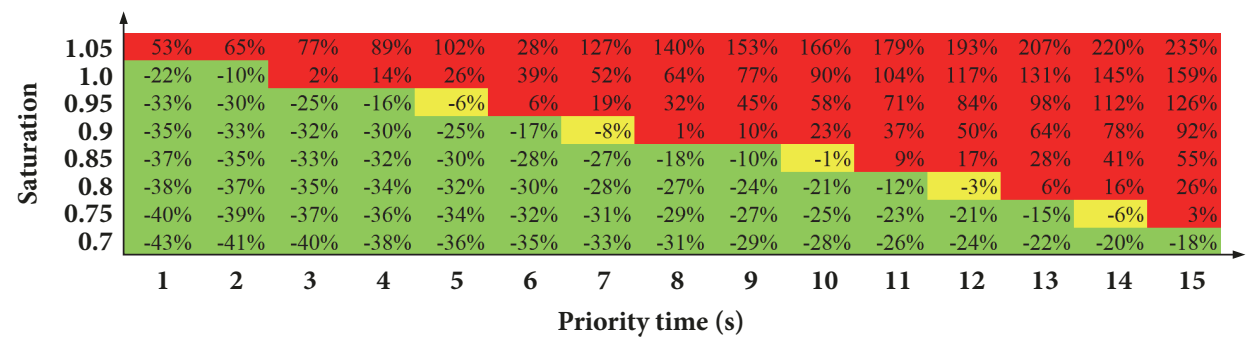

FIGURE 14: Table with recommended values for bus signal priorities.

Simple distribution of the added red-light time to the following phases according to the phase splits or resplitting the green light time period according to the degree of saturation was shown to be unsuitable.

(3) A table with recommended bus priority values was presented according to the saturation of the intersection and the length of the priority time. More than $6 \%$ reductions in person delay can be obtained for undersaturated intersections when the priority time was less than $5 \mathrm{~s}$. Moreover, even when the intersection saturation was 0.95 , the bus signal priority could be applied if only the priority time was less than $5 \mathrm{~s}$.

The signal control model proposed in this study can be applied in near-saturated intersection. However, the issue on the multiple bus requests was not discussed and constitutes a research topic for our ongoing and future research endeavors.

\section{Data Availability}

The data used to support the findings of this study are available from the corresponding author upon request.

\section{Conflicts of Interest}

The authors declare that they have no conflicts of interest.

\section{Acknowledgments}

The research is supported by the National Natural Science Foundation of China under Grant no. 51608324.

\section{References}

[1] J. Shi, Z. Wu, and J. Jin, "Reform Beijing to a public transit oriented city - From the view of transportation equity," Journal of Advanced Transportation, vol. 45, no. 2, pp. 96-106, 2011.

[2] K. M. N. Habib, L. Kattan, and T. Islam, "Model of personal attitudes towards transit service quality," Journal of Advanced Transportation, vol. 45, no. 4, pp. 271-285, 2011.

[3] M. Eichler and C. F. Daganzo, "Bus lanes with intermittent priority: strategy formulae and an evaluation," Transportation Research Part B: Methodological, vol. 40, no. 9, pp. 731-744, 2006.

[4] W. J. Ma, K. L. Head, and Y. H. Feng, "Integrated optimization of transit priority operation at isolated intersections: a person-capacity-based approach," Transportation Research Part C: Emerging Technologies, vol. 40, pp. 49-62, 2014.

[5] M. Mesbah, M. Sarvi, and G. Currie, "New methodology for optimizing transit priority at the network level," Transportation Research Record, vol. 2089, no. 12, pp. 93-100, 2008. 
[6] S. I. Guler and M. J. Cassidy, "Strategies for sharing bottleneck capacity among buses and cars," Transportation Research Part B: Methodological, vol. 46, no. 10, pp. 1334-1345, 2012.

[7] A. H. Alomari, H. Al-Deek, A. Sandt, J. H. Rogers, and O. Hussain, "Regional evaluation of bus rapid transit with and without transit signal priority," Transportation Research Record, vol. 2554, no. 6, pp. 46-59, 2016.

[8] K. N. Balke, C. L. Dudek, and T. Urbanik II, "Development and evaluation of intelligent bus priority concept," Transportation Research Record, vol. 1727, no. 1543, pp. 12-19, 2000.

[9] A. Skabardonis, "Control strategies for transit priority," Transportation Research Record, vol. 1727, no. 1584, pp. 20-26, 2000.

[10] M. Estrada, C. Trapote, M. Roca-Riu, and F. Robusté, "Improving bus travel times with passive traffic signal coordination," Transportation Research Record, vol. 2111, no. 9, pp. 68-75, 2009.

[11] J. Lee, A. Shalaby, J. Greenough, M. Bowie, and S. Hung, "Advanced transit signal priority control with online microsimulation-based transit prediction model," Transportation Research Record, vol. 1925, no. 1, pp. 185-194, 2005.

[12] V. Ngan, T. Sayed, and A. Abdelfatah, "Impacts of various parameters on transit signal priority effectiveness," Journal of Public Transportation, vol. 7, no. 3, pp. 71-93, 2004.

[13] J. Jacobson and Y. Sheffi, "Analytical model of traffic delays under bus signal preemption: theory and application," Transportation Research Part B: Methodological, vol. 15, no. 2, pp. 127138, 1981.

[14] P. G. Furth and T. H. J. Muller, "Conditional bus priority at signalized intersections: better service with less traffic disruption," Transportation Research Record, vol. 1731, no. 1, pp. 23-30, 2000.

[15] Z. R. Abdy and B. R. Hellinga, "Analytical method for estimating the impact of transit signal priority on vehicle delay," Journal of Transportation Engineering, vol. 137, no. 8, pp. 589-600, 2011.

[16] K. Ling and A. Shalaby, "Automated transit headway control via adaptive signal priority," Journal of Advanced Transportation, vol. 38, no. 1, pp. 45-67, 2003.

[17] E. Christofa and A. Skabardonis, "Traffic signal optimization with application of transit signal priority to an isolated intersection," Transportation Research Record, vol. 2259, no. 1, pp. 192201, 2011.

[18] L. Head, D. Gettman, and W. Zhiping, "Decision model for priority control of traffic signals," Transportation Research Record, vol. 1978, no. 1, pp. 169-177, 2006.

[19] W. Ma, X. Yang, and Y. Liu, "Development and evaluation of a coordinated and conditional bus priority approach," Transportation Research Record, vol. 2145, no. 6, pp. 49-58, 2010.

[20] E. Christofa, K. Ampountolas, and A. Skabardonis, "Arterial traffic signal optimization: a person-based approach," Transportation Research Part C: Emerging Technologies, vol. 66, pp. 27-47, 2016.

[21] W. Ma, Y. Liu, and X. Yang, "A dynamic programming approach for optimal signal priority control upon multiple highfrequency bus requests," Journal of Intelligent Transportation Systems: Technology, Planning, and Operations, vol. 17, no. 4, pp. 282-293, 2013.

[22] J. Hu, B. B. Park, and Y.-J. Lee, "Coordinated transit signal priority supporting transit progression under connected vehicle technology," Transportation Research Part C: Emerging Technologies, vol. 55, pp. 393-408, 2015.

[23] M. Li, Y. Yin, W.-B. Zhang, K. Zhou, and H. Nakamura, "Modeling and implementation of adaptive transit signal priority on actuated control systems," Computer-Aided Civil and Infrastructure Engineering, vol. 26, no. 4, pp. 270-284, 2011.
[24] Q. He, K. L. Head, and J. Ding, "Multi-modal traffic signal control with priority, signal actuation and coordination," Transportation Research Part C: Emerging Technologies, vol. 46, pp. 65-82, 2014.

[25] Y. J. Lin, X. F. Yang, G.-L. Chang, and N. Zou, "Transit priority strategies for multiple routes under headway-based operations," Transportation Research Record, vol. 2356, no. 5, pp. 34-43, 2013.

[26] S. Z. Altun and P. G. Furth, "Scheduling buses to take advantage of transit signal priority," Transportation Research Record, vol. 2111, no. 7, pp. 50-59, 2009.

[27] S. I. Guler, V. V. Gayah, and M. Menendez, "Bus priority at signalized intersections with single-lane approaches: A novel presignal strategy," Transportation Research Part C: Emerging Technologies, vol. 63, pp. 51-70, 2016.

[28] J. Stevanovic, A. Stevanovic, P. T. Martin, and T. Bauer, "Stochastic optimization of traffic control and transit priority settings in VISSIM," Transportation Research Part C: Emerging Technologies, vol. 16, no. 3, pp. 332-349, 2008.

[29] W. Ma, Y. Liu, and B. Han, "A rule-based model for integrated operation of bus priority signal timings and traveling speed," Journal of Advanced Transportation, vol. 47, no. 3, pp. 369-383, 2013.

[30] F. Dion and B. Hellinga, "A rule-based real-time traffic responsive signal control system with transit priority: application to an isolated intersection," Transportation Research Part B: Methodological, vol. 36, no. 4, pp. 325-343, 2002.

[31] M. Janos and P. G. Furth, "Bus priority with highly interruptible traffic signal control: simulation of San Juan's Avenida Ponce de Leon," Transportation Research Record, vol. 1811, no. 1, pp. 157165, 2002.

[32] J. Hu, B. Park, and A. E. Parkany, “Transit signal priority with connected vehicle technology," Transportation Research Record, vol. 2418, no. 3, pp. 20-29, 2014.

[33] J. Li, W. Wang, H. J. van Zuylen, N. N. Sze, X. W. Chen, and H. Wang, "Predictive strategy for transit signal priority at fixedtime signalized intersections case study in Nanjing, China," Transportation Research Record, vol. 2311, no. 12, pp. 124-131, 2012.

[34] Transportation Research Board, "Highway capacity manual 2010. Washington D C: National Research Council," 2010. 


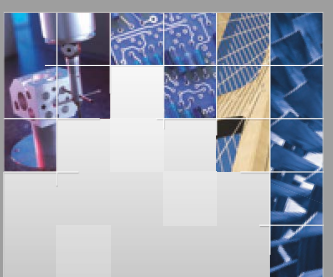

\section{Enfincering}
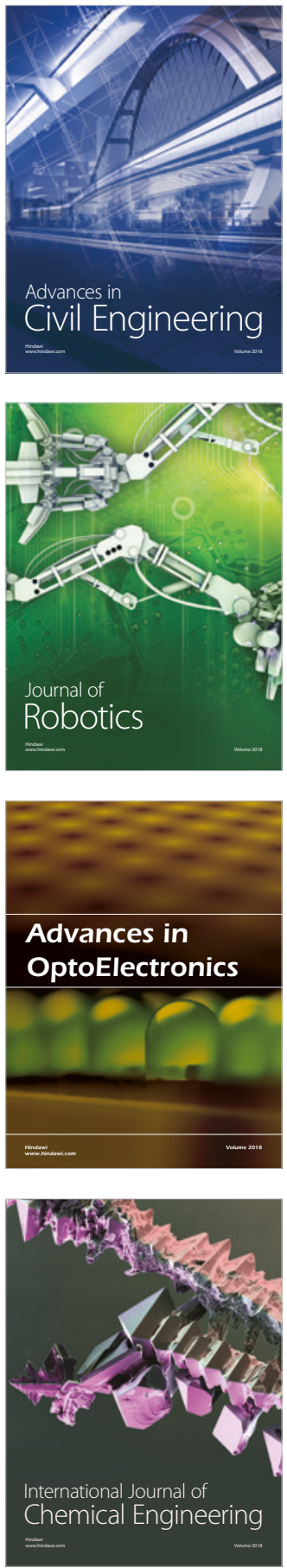

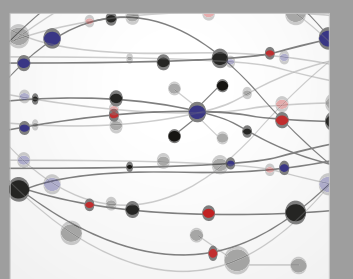

\section{Rotating \\ Machinery}

The Scientific World Journal

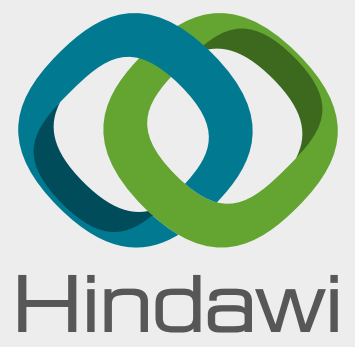

Submit your manuscripts at

www.hindawi.com
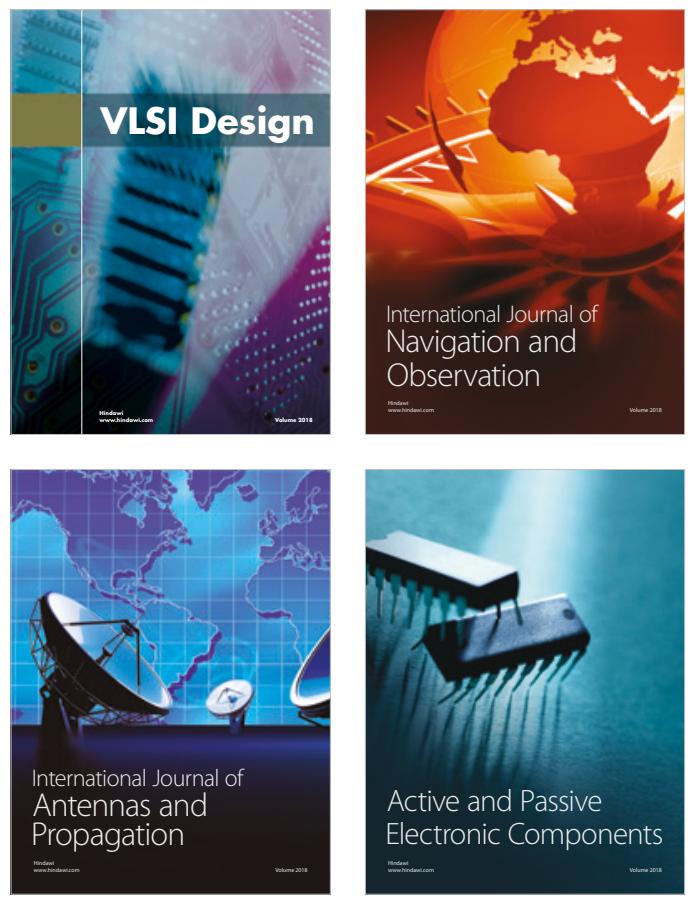
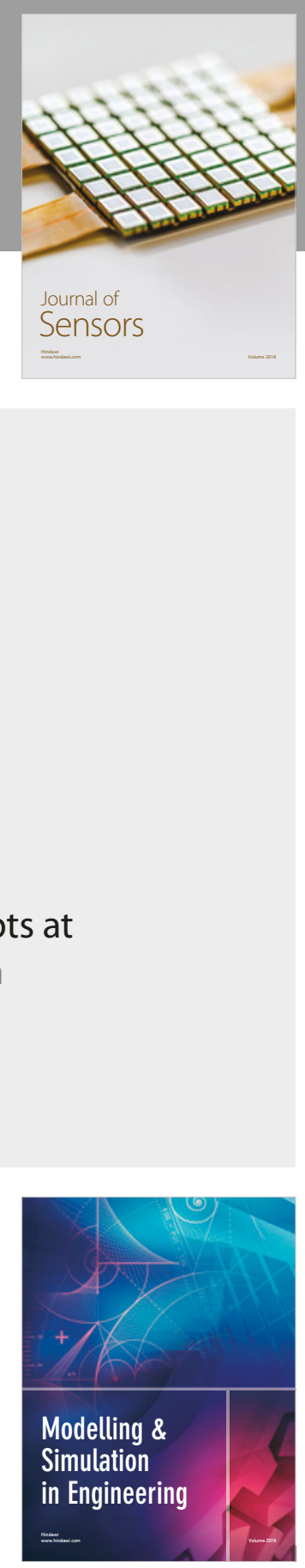

\section{Advances \\ Multimedia}
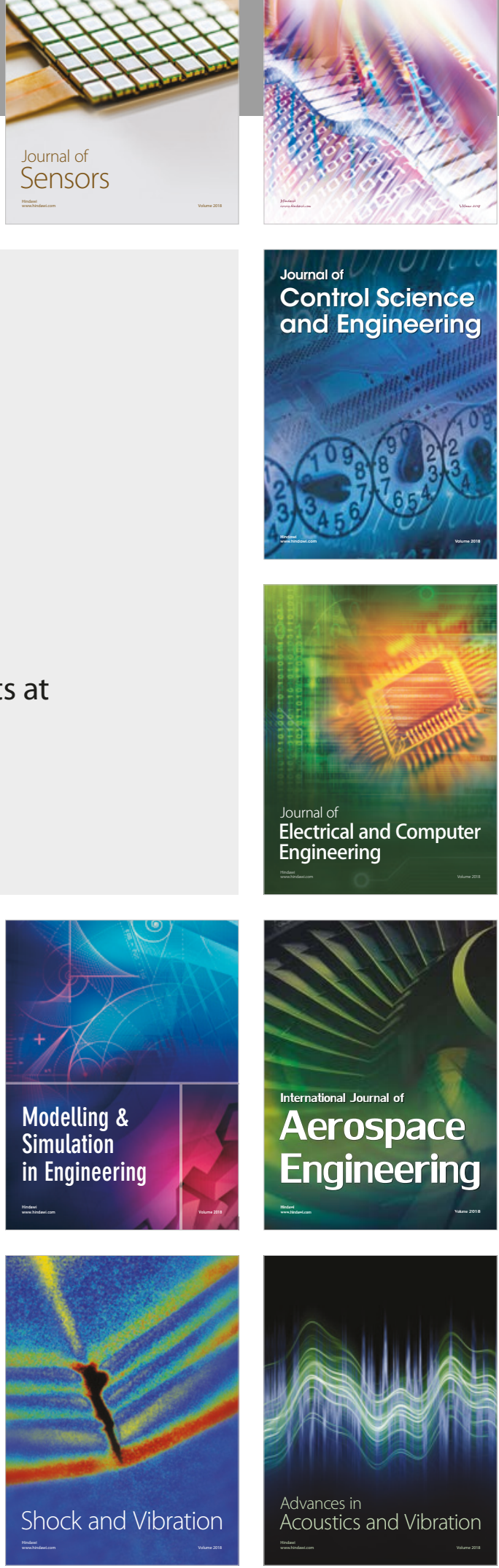